\title{
Erosive pustular dermatosis
}

\author{
Christopher Sladden MB BCh, Rebecca Afford BSc
}

- Cite as: CMAJ 2019 June 17;191:E667. doi: 10.1503/cmaj.181685

A 73-year-old man presented to a dermatology clinic with a $10 \times 9 \mathrm{~cm}$ erosive plaque covered with a thick adherent yellow-brown crust of the scalp (Figure 1). The mass had been enlarging for more than 3 years, without diagnosis or treatment. The patient's history included tinea capitis at age 8 years, treated with radiotherapy (the historical gold standard treatment), and multiple nonmelanoma skin cancers of the head, which had been treated surgically. Removal of the crust revealed a friable, eroded, tender, somewhat vascular plaque. The differential diagnosis included squamous cell carcinoma; other nonmelanoma skin cancers; inflammatory dermatoses, including pemphigus vegetans and Brunsting-Perry type pemphigoid; kerion, pyoderma gangrenosum; and erosive pustular dermatosis. Bacterial skin swabs were negative. Potassium hydroxide and dermatophyte fungal cultures were negative, as was rapid plasma reagin testing. Three punch biopsies from representative areas were taken for histopathologic evaluation, including direct immunofluorescence studies. Histologic findings of fibrosis; chronic mixed inflammation, including plasma cell aggregation; reactive epidermal cytological atypia; serum crust; hyperkeratosis; and parakeratosis were noted on all specimens. There were no findings of malignancy. Histopathology was compatible with erosive pustular dermatosis.

Erosive pustular dermatosis has a striking clinical presentation and may be mistaken for cutaneous malignancy. Clinicopathologic correlation is critical because histopathology is nonspecific; thus, erosive pustular dermatosis is a diagnosis of exclusion supported by a positive response to treatment. ${ }^{1}$ It is a noninfectious inflammation of the skin, generally the scalp or legs, associated with chronic ultraviolet damage (in this case, previous radiotherapy may have been involved) and subsequent skin trauma. ${ }^{2}$

Systemic antibiotics (to treat any associated infection) and corticosteroids may be required; ${ }^{3}$ however, in this patient, topical treatment with clobetasol $0.05 \%$ and fusidic acid $2 \%$ ointments (1:1) used twice daily for 1 month produced excellent improvement (Figure 2). This was followed by hydrocortisone acetate $2.5 \%$ and fusidic acid $2 \%$ ointments (1:1) applied 3 times daily to complete resolution over the following 2 months.

\section{References}

1. Wilk M, Zelger BG, Hauser U, et al. Erosive pustular dermatosis of the scalp: reappraisal of an underrecognized entity. J Dtsch Dermatol Ges 2018;16:15-9.

2. Tomasini C, Michelerio A. Erosive pustular dermatosis of the scalp: a neutrophilic folliculitis within the spectrum of neutrophilic dermatoses: a clinicopathologic study of thirty cases. J Am Acad Dermatol 2018 Oct 25. pii: S0190-9622(18)32797-X. [Epub ahead of print].

3. Brouard MC, Prins C, Chavaz $\mathrm{P}$, et al. Erosive pustular dermatosis of the leg: report of three cases. Br J Dermatol 2002;147:765-9.

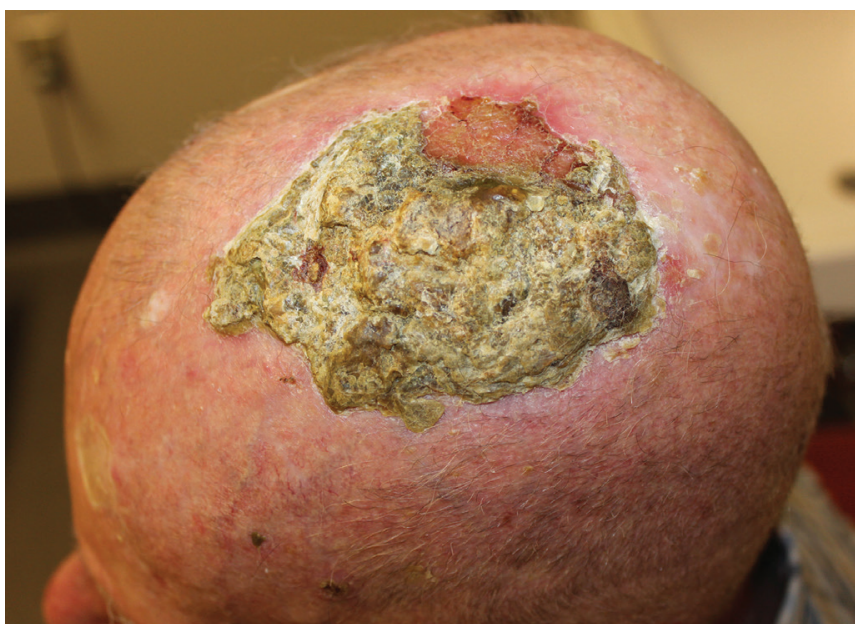

Figure 1: Erosive plaque covered with a thick adherent crust of the scalp in a 73-year-old man, consistent with erosive pustular dermatosis.

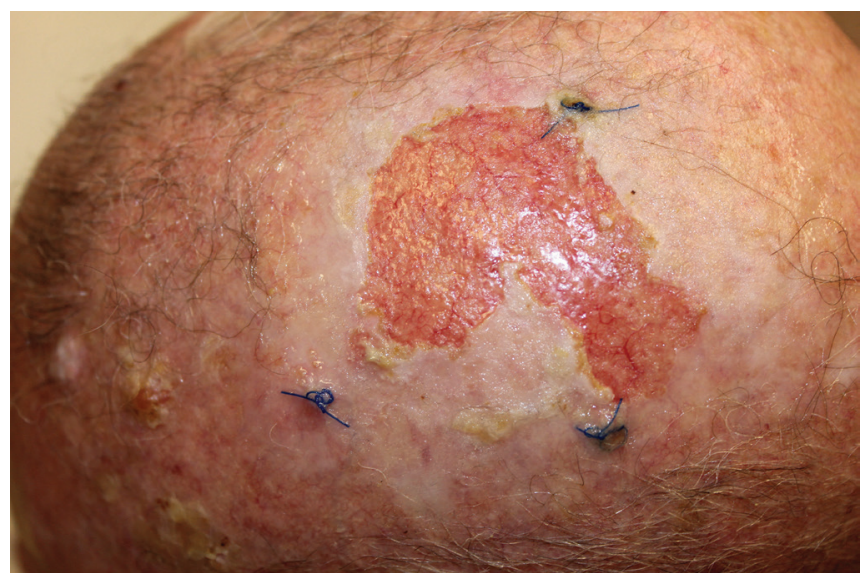

Figure 2: The patient's scalp after 1 month of treatment with clobetasol $0.05 \%$ and fusidic acid $2 \%$ ointment applied twice daily. The patient did not follow written instructions for suture removal; however, the sutures serve as a useful marker for re-epithelialization.

\section{Competing interests: None declared.}

This article has been peer reviewed.

The authors have obtained patient consent.

Affiliations: Department of Dermatology and Skin Science (Sladden), Faculty of Medicine; Department of Medicine (Afford), University of British Columbia, Vancouver, BC

Correspondence to: Rebecca Afford, r.afford@alumni.ubc.ca 